\title{
Periodic Structure towards the Terahertz Region Manufactured Using High Resolution 3D Printing
}

\author{
A. R. Phipps ${ }^{1}$, A. J. MacLachlan ${ }^{1}$, L. Zhang ${ }^{1}$, C. W. Robertson ${ }^{1}$, I. V. Konoplev ${ }^{2}$, A. D. R. Phelps ${ }^{1}$ and A. W. Cross ${ }^{1}$ \\ ${ }^{1}$ Department of Physics, SUPA, University of Strathclyde, Glasgow, G4 0NG, Scotland, UK. \\ ${ }^{2}$ JAI, Department of Physics, University of Oxford, Oxford, OX1 3RH, England, UK.
}

\begin{abstract}
Periodic structures used for high power millimetre and sub-millimetre sources that implement relativistic beam wave interactions have historically involved the implantation of a dielectric layer around the inner wall of the interaction region or a periodic corrugated structure that serves to reduce the velocity of an internal electromagnetic wave. Moving towards the $\mathrm{THz}$ regime, the physical dimensions required to manufacture such a cavity become prohibitively difficult. Ongoing attempts to develop manufacturing processes that provide higher resolution have resulted in a number of viable techniques. Additive Manufacturing or 3D printing offers the possibility of producing components on this scale quickly and efficiently. Here $0.1,0.4$ and $1 \mathrm{THz}$ periodic structures are realised using high resolution (16 micron) 3D printing technology.
\end{abstract}

Keywords-3D printing, Periodic Surface Lattice, Terahertz radiation

\section{INTRODUCTION}

Vacuum electronics has provided a means by which to produce high power radiation in the millimetre and submillimetre range [1]. Subsequent development of new and novel ways of promoting energy exchange from relativistic electrons beams to spatially confined electromagnetic fields has allowed MW output powers at frequencies of $\sim 35 \mathrm{GHz}$ [2]. Ka-band (26.5-40) $\mathrm{GHz}$ and W-band (75-110) $\mathrm{GHz}$ structures with $\mathrm{D} / \lambda \approx 7$ (where $\mathrm{D}$ is the aperture diameter and $\lambda$ the wavelength) were both manufactured by milling sinusoidal perturbations on the outer wall of an aluminium former and electrodepositing copper directly onto the surface $[3,4]$. The aluminium was then dissolved in a strong alkaline solution, leaving the copper PSL structure with the perturbations on the inner surface. Moving from Ka-band to W-band was possible as the perturbation amplitude of 400 microns was not limited by the manufacturing tolerances of the HAAS TM-1CE milling machine $+-5 \mu \mathrm{m}$ (maximum tool speed of 4,000 revolutions per minute). Upgrading the HAAS TM-1CE milling machine with installation of high speed compressed air driven tooling for use with smaller sized tools with speeds of up to 50,000 revolutions per minute would enable even higher tolerances to be achieved.

A 2D Periodic Surface Lattice (PSL) was successfully created using a conventional mechanical mill to manufacture the aluminium former with the copper electro-deposited on the aluminium and then dissolved away. Good agreement between theoretical predictions and experimental measurement of the transmission properties of the 2D PSL using this manufacturing technique was achieved [5]. It became clear however, when moving beyond $\mathrm{W}$-band and certainly into the $\mathrm{THz}$ region where perturbation amplitudes would potentially be on the micron scale a more accurate higher performance mill [6] or a new manufacturing technique would be required. 3D printing represents the next stage of an ongoing process where smaller and smaller components can be manufactured with increasing precision. Now, at this early stage, the 3D printing process offers the possibility of producing objects that have resolutions on the 16 micron scale. The tolerances achievable with $3 \mathrm{D}$ printing are projected to decrease over time as the technology develops resulting in a higher degree of accuracy.

\section{RESULTS}

3D printing was originally developed in the mid 1980's [7]. 3D printing of structures such as those modelled in fig. 1 is an additive process by which consecutive layers in the $\mathrm{x}-\mathrm{Z}$ plane are deposited sequentially in the +ve $y$ direction resulting in the $2 \mathrm{D}$ PSLs shown in fig. 2 . The printing process follows the pattern in a given CAD input file, usually in the STL (Stereolithograph) file format where every face is built from a series of interconnected triangles represented by 3 separate 32-bit floating-point Cartesian coordinates. The physical parameters for three separate 2D PSLs are shown in tables 1,2 and 3 . Maintaining $\mathrm{D} / \lambda \approx 7$ these structures have an operating frequency of $0.1,0.4$ and $1 \mathrm{THz}$ and have each been produced by the same manufacturing process with the exception of the $0.1 \mathrm{THz}$ PSL which differs in that the original shape was 3D printed in wax and the finished part was created from a mold of that former. More often now the new X3D file format is implemented which incorporates the XML programming interface and further enhancements over its predecessors.

Table 1 The physical parameters for the $0.1 \mathrm{THz} 2 \mathrm{D}$ PSL.

\begin{tabular}{|l|c|c|}
\hline Longitudinal Period & $\mathrm{dz}$ & $3 \mathrm{~mm}$ \\
\hline Azimuthal Variations & $\mathrm{m}$ & 20 \\
\hline Number of Longitudinal Periods & $\mathrm{x}$ & 7 \\
\hline Perturbation Amplitude & $\mathrm{dr}$ & $400 \mathrm{microns}$ \\
\hline Unperturbed Inner Radius & $\mathrm{r}$ & $10 \mathrm{~mm}$ \\
\hline
\end{tabular}


Author Accepted Manuscript: $8^{\text {th }}$ UCMMT 2015, 14-15 September 2015, Cardiff, UK.

Published online in IEEE Xplore 28 April 2016

Table 2 The physical parameters for the $0.4 \mathrm{THz} 2 \mathrm{D}$ PSL.

\begin{tabular}{|l|c|c|}
\hline Longitudinal Period & $\mathrm{Dz}$ & $0.75 \mathrm{~mm}$ \\
\hline Azimuthal Variations & $\mathrm{M}$ & 20 \\
\hline Number of Longitudinal Periods & $\mathrm{X}$ & 7 \\
\hline Perturbation Amplitude & $\mathrm{Dr}$ & 100 microns \\
\hline Unperturbed Inner Radius & $\mathrm{R}$ & $2.5 \mathrm{~mm}$ \\
\hline
\end{tabular}

Table 3 The physical parameters for the $1 \mathrm{THz} 2 \mathrm{D}$ PSL.

\begin{tabular}{|l|c|c|}
\hline Longitudinal Period & $\mathrm{dz}$ & $0.3 \mathrm{~mm}$ \\
\hline Azimuthal Variations & $\mathrm{M}$ & 20 \\
\hline Number of Longitudinal Periods & $\mathrm{X}$ & 7 \\
\hline Perturbation Amplitude & $\mathrm{dr}$ & 40 microns \\
\hline Unperturbed Inner Radius & $\mathrm{R}$ & $1 \mathrm{~mm}$ \\
\hline
\end{tabular}

The design is sliced into digital layers so that a curve is 'approximated' by many square-sided slices, with the thickness of each layer representing the resolution of that particular 3D printing process. At $0.1 \mathrm{THz}$ the perturbation amplitude is 400 microns in size. Representation of this structure with layers of 16 microns results in a relatively high level of accuracy and the finished component appears to hold a high level of detail (fig. 2a). In the case of this specific piece (silver $0.1 \mathrm{THz} 2 \mathrm{D}$ PSL) the complete part was printed in wax and this former was used to create a mold in which a molten silver (92.5\%) chromium $(7.5 \%)$ alloy was set. The resultant component seen in fig. $2 \mathrm{a}$ was successfully incorporated in a cold test system with the transmission of millimetre waves measured using a Vector Network Analyzer. The on axis frequency sweep for this device is seen in fig. $2 b$, here, the cavity formation process means that a portion of the electric field stays within the PSL structure to form the cavity, due to this fact there is a dip in the output frequency spectrum. The blue line represents the experimental observation of this process for a PSL with perturbation amplitude of 400 microns, whereas the green and red lines represent PSLs with a perturbation amplitude of 400 and 450 microns respectively as seen in CST MWS simulations. This discrepancy between the experimental and numerical results is due to the fact that the perturbations in the manufactured component do not have an amplitude of exactly 400 microns but in fact there will be a distribution of amplitudes that equal $(425 \pm 16)$ microns. The tolerance in the period of the $400 \mathrm{GHz} 2 \mathrm{D}$ PSL from the values quoted by the manufacturer was $(750 \pm 16)$ microns. At $0.4 \mathrm{THz}$ the perturbation amplitude would be 100 microns, so with a resolution of 16 microns there would be 6 layers available to represent the amplitude of the perturbation of the PSL. Fig. 2c demonstrates the result of the $3 \mathrm{D}$ printing process with a $2 \mathrm{D}$ PSL former printed in VisiJet Crystal, EX 200 Plastic. This former represents the physical space from within the PSL structure and will be coated in a chromium adhesive layer approximately 1 micron thick upon which copper will be electrodeposited. The on-axis frequency sweep seen in a CST MWS simulation can be viewed in fig. $2 \mathrm{~d}$, at $0.4 \mathrm{THz}$ the dip in the graph appears to be far wider than the model, ranging from approximately $0.36 \mathrm{THz}$ to $0.42 \mathrm{THz}$ giving a bandwidth of 60 $\mathrm{GHz}$. Moving to $1 \mathrm{THz}$ the accuracy of the current 3D printing process decreases as a single perturbation structure is represented by only 3 layers of additive material. Fig. 2e displays the resultant structure as seen using a scanning electron microscope. Fig. $2 \mathrm{f}$ demonstrates the on axis frequency sweep for the $1 \mathrm{THz} 2 \mathrm{D}$ PSL as simulated using CST Microwave Studio and it can be seen it has a frequency centered at $0.975 \mathrm{THz}$ with a frequency range $0.85 \mathrm{THz}$ to 1.2 THz, (-10 dB point).

The 2D PSL is designed as an interaction region for the formation of a cavity eigenmode that is the resultant field distribution of a $\mathrm{TM}_{0, \mathrm{n}}$ Volume Field (VF) and an induced $\mathrm{HE}_{7,1}$ Surface Field (SF). This interaction takes place within the 2D PSL region and becomes the basis for a novel type of high power Backward Wave Oscillator (BWO) that utilizes the Cherenkov instability. The actual modes involved and the resultant field can be visualized in ATK's MAGIC 3D and a relevant example of which is seen below in fig. 1 .
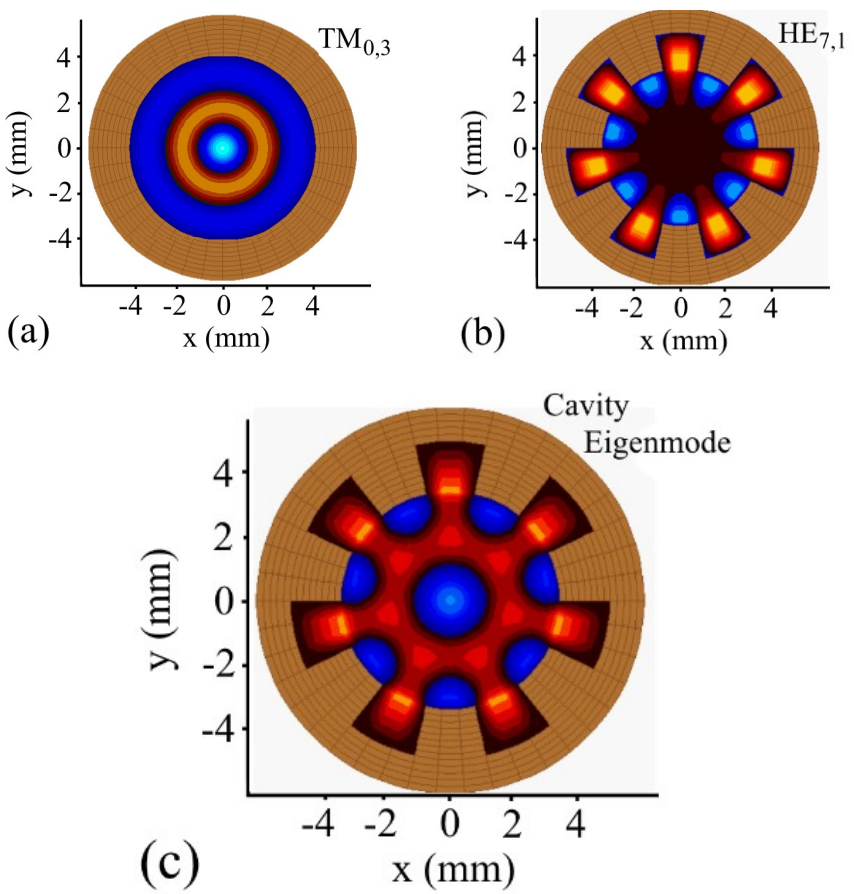

Figure 1: These images demonstrate the VF/SF coupling within the PSL region as seen in MAGIC 3D for a 2D PSL of $\mathrm{r}=4 \mathrm{~mm}, \mathrm{dr}=0.8 \mathrm{~mm}$ and $\mathrm{dz}=1.6$ mm. Image (a) displays the $\mathrm{TM}_{0,3} \mathrm{VF}$, image (b) demonstrates the $\mathrm{HE}_{7,1} \mathrm{SF}$ and image (c) shows the resultant cavity eigenmode seen within the structure.

The 2D PSL is ultimately a mode selection cavity structure 
Author Accepted Manuscript: $8^{\text {th }}$ UCMMT 2015, 14-15 September 2015, Cardiff, UK.

Published online in IEEE Xplore 28 April 2016

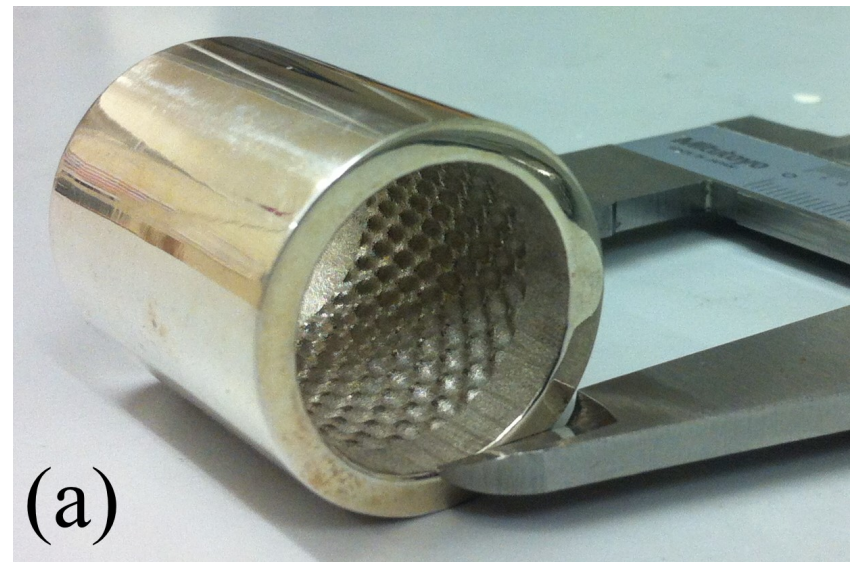

2D PSL Farfield Frequency Sweep

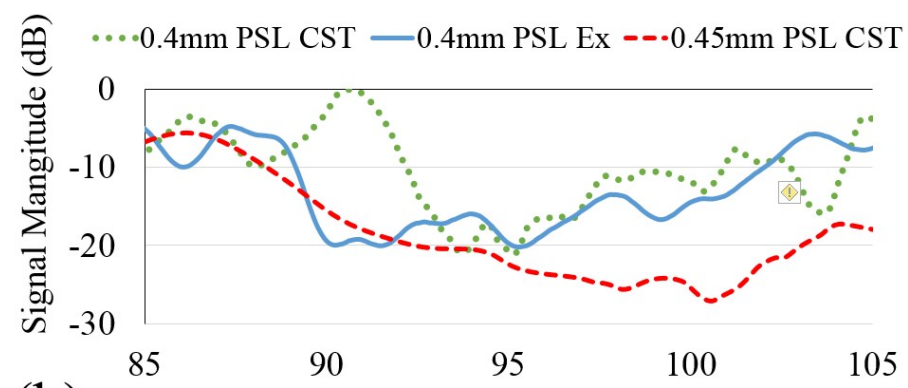

(b) W-Band Frequency (GHz)

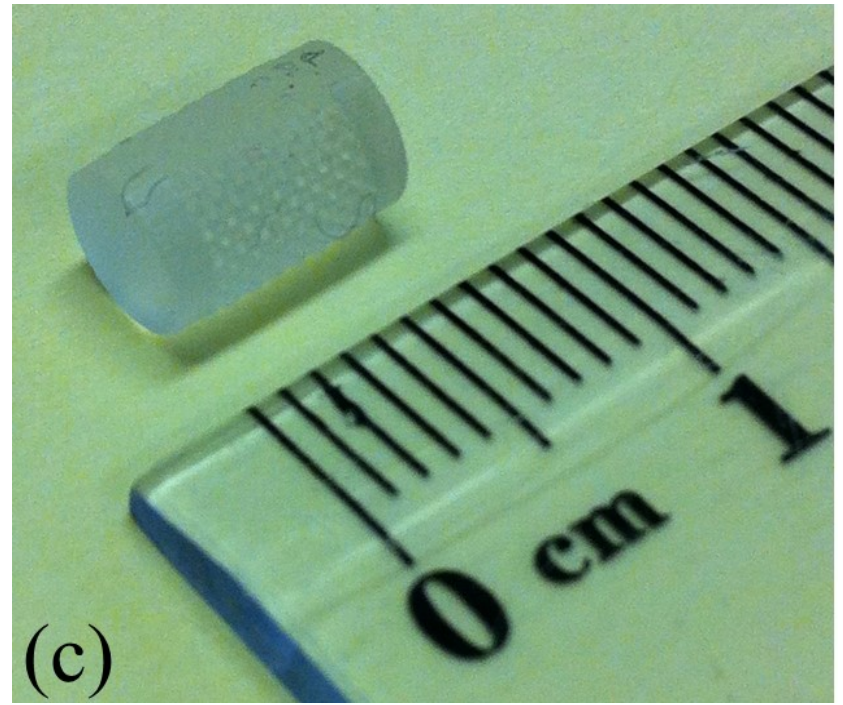

$400 \mathrm{GHz} 2 \mathrm{D}$ PSL Frequency Sweep
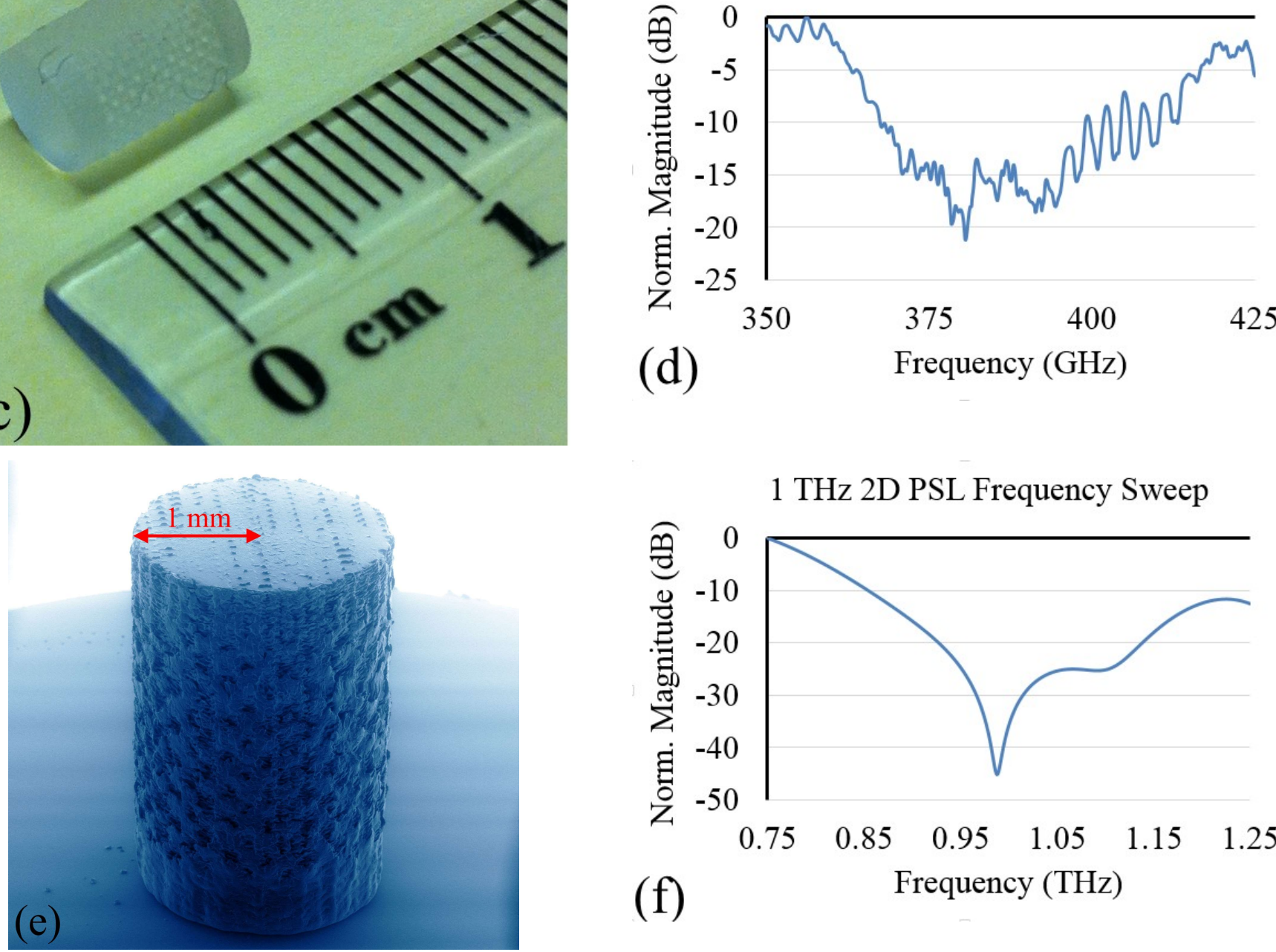

$1 \mathrm{THz} 2 \mathrm{D}$ PSL Frequency Sweep

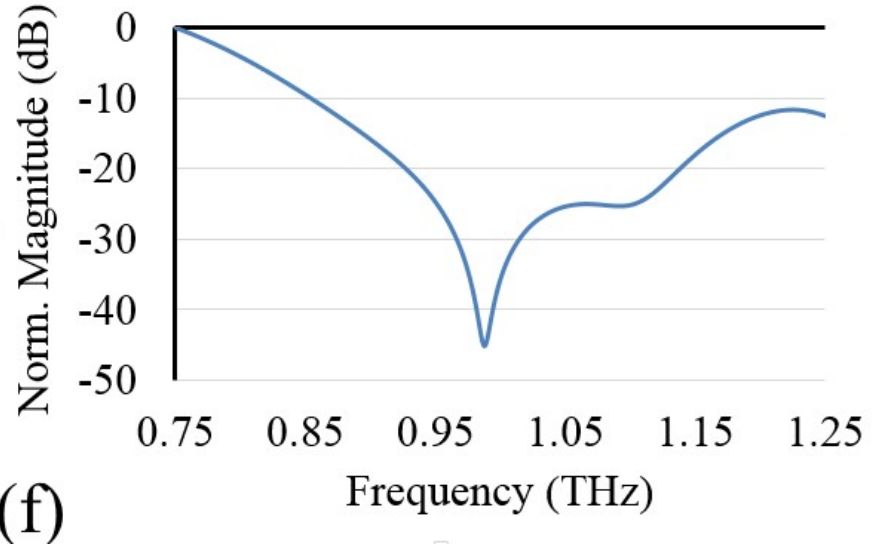

Figure 2: (a) The $0.1 \mathrm{THz} 2 \mathrm{D}$ PSL with a longitudinal period of $3 \mathrm{~mm}$ resulting in a total length of $31 \mathrm{~mm}$. Made from a silver chromium alloy using the $3 \mathrm{D}$ printing process. (b) The on-axis frequency sweep for the $0.1 \mathrm{THz} 2 \mathrm{D}$ PSL. The blue line represents the experimental measurements and the other two lines represent the simulations of the structure, one with a perturbation amplitude of $0.4 \mathrm{~mm}$ (green dotted line) and one with $0.45 \mathrm{~mm}$ (red dashed line). (c) The $0.4 \mathrm{THz} 2 \mathrm{D}$ PSL has 7 longitudinal periods of $\mathrm{dz}=0.75 \mathrm{~mm}$ and 20 azimuthal variations, with an unperturbed radius of $2.5 \mathrm{~mm}$. (d) In this simulation the dip in the graph represents a point of resonance for the field within the cavity region and the cavity forms at this frequency. (e) The $1 \mathrm{THz} 2 \mathrm{D}$ PSL has a longitudinal period of $0.3 \mathrm{~mm}$ resulting in a total length of $3.1 \mathrm{~mm}$. (f) In this simulation the dip here is much wider than in the previous two spectrums and is centered at $\sim 0.975 \mathrm{THz}$. 
that utilizes the structural design of the cavity region to induce the formation of a particular cavity mode from a Surface Field (SF) that can then be superimposed onto the existing volume field (VF) with the resulting cavity eigenmode available for use in future experimentation. The images seen in fig. 1 represent the different stages of a particular interaction process with image (a) demonstrating the $\mathrm{TM}_{0,3}$ volume field that enters the cavity region and is scattered at the perturbations. Fig. 1b represents the surface field $\mathrm{HE}_{7,1}$ mode that is seen at the perturbations and fig. $1 \mathrm{c}$ is the cavity eigenmode structure that is formed from the superposition of the surface field and the volume field. This final field distribution is used in a Cherenkov instability interaction to produce high microwave output at the operating frequency of the device. The tailoring of the 2D PSL structure's physical dimensions allows the formation of the cavity eigenmode from the desired input fields. In particular the unperturbed inner radius forces the $\mathrm{TM}_{0,3}$ mode to be near cut-off and so it will experience strong reflection at the perturbations. Other unwanted $\mathrm{TM}_{0, \mathrm{n}}$ modes of lower radial index will pass through the interaction region and although they may interact with the cavity eigenmode they will not experience sufficient reflection to form a cavity and so will pass out of the exit aperture after a single pass. Numerical simulations conducted in MAGIC 3D confirm the successful formation of the cavity eigenmode from the $\mathrm{TM}_{0,3}$ and $\mathrm{HE}_{7,1}$ modes.

\section{SUMMARY}

This paper demonstrates that the additive manufacturing process is now becoming relevant for many applications where standard engineering processes are becoming challenging and time consuming. 3D printing offers a viable and novel way of producing small scale components quickly and efficiently and as the technology develops the level of accuracy and detail that this process offers will increase. As we move into the $\mathrm{THz}$ regime this technology may become a useful tool in the creation of components and structures requiring high resolution and small fabrication tolerances.

\section{ACKNOWLEDGMENT}

This work is sponsored by the Engineering and Physical Sciences Research Council (EPSRC), UK. The authors thank David Barclay for assistance in the manufacture of the components used in the VNA measurement system.

\section{REFERENCES}

[1]. N. S. Ginzburg, N. Yu. Peskov, A. S. Sergeev, "Two-dimensional double-periodic Bragg resonators for free-electron lasers", Opt. Commun., vol. 96, pp. 254-258, Feb. 1993.

[2]. I. V. Konoplev, P. McGrane, W. He, A. W. Cross, A. D. R. Phelps, C. G. Whyte, K. Ronald, C. W. Robertson, "Experimental study of coaxial free-electron maser based on two-dimensional distributed feedback”, Phys. Rev. Lett., vol 96, 035002, Jan. 2006.

[3]. I. V. Konoplev, A. J. MacLachlan, C. W. Robertson, A. W. Cross and A. D. R. Phelps, "Cylindrical periodic surface lattice as a metadielectric: Concept of a surface-field Cherenkov source of coherent radiation", Phys. Rev. A, vol. 84, 013826, July 2011.

[4]. I. V. Konoplev, A. R. Phipps, A. D. R. Phelps, C. W. Robertson, K. Ronald, A. W. Cross, "Surface field excitation by an obliquely incident wave", Appl. Phys. Lett., vol 102, 141106, Apr. 2013.

[5]. I. V. Konoplev, L. Fisher, A. W. Cross, A. D. R. Phelps, K. Ronald and C. W. Robertson, "Surface wave Cherenkov maser based on a periodic lattice”, Appl. Phys. Lett., vol. 96, 261101, June 2010.

[6]. http://www.ralspace.stfc.ac.uk/ralspace/11282.aspx 19/08/2015

[7]. C. W. Hull, "Apparatus for Production of Three-Dimensional Objects by Stereolithography", U.S. Patent 4,575,330 